\title{
Contextuality and Dynamics
}

\author{
Theodor Harder
}

Universität Bielefeld, Fakultät für Soziologie

\section{Kontextualität und Dynamik}

Inhalt: Die methodologischen Probleme der Mehr-Ebenen-Analyse werden von einem elementaren statistischen Standpunkt aus entwickelt. Es gibt verschiedene Dimensionstypen wie z.B. die Zeit, die ,stimultan“ Individuen oder regionalen Einheiten als Stichproben-Realisation zugeordnet werden können, also Dimensionen, die unabhängig von der Dimension „Zeit" sind. Dagegen sind verschiedene Kollektivitäts-Ebenen keine unabhängigen Dimensionen. Ein Individuum, das zu genau einem Kontext gehört, kann nicht über mehrere Kontexte hinweg konstant gehalten werden. Dieses Hindernis kann dadurch überwunden werden, daß man statt mit Individuen mit Typen arbeitet. Ein solcher statischer Modell-Ansatz ist aber theoretisch unbefriedigend. Das Problem der Kontext-Variation fordert einen dynamischen Ansatz heraus. Die dazu gehörenden Daten-Arten wie Zeitreihen, biographische und historische Quellen, werfen von der Erhebung und Analyse her zusätzliche Probleme auf. Ein zentrales Problem, dessen Behandlung von einem dynamischen Mehr-Ebenen-Ansatz profitieren würde, ist die Modellierung von Mobilitätstheorien, wobei die Identität dessen, der seine Position wechselt, explizit berücksichtigt würde.

Abstract: The methodological problems of multi-level analysis are developed from an elementary statistical standpoint. There are different types of dimensions as f.i. time which can be associated with any individual or regional sampling realization while different levels of collectivity are not independent dimensions. An individual belonging to just one context cannot be kept constant across different contexts. Working with types instead of individuals makes up for this deficiency. However, the static model is theoretically unsatisfactory and calls for a dynamic approach. Data like time series, biographical documents and historical sources would have to be collected and integrated. A special field of application would be mobility models with emphasis on the identity problem of social movers.

There is no doubt that micro-macro analogies are at least as old as Plato's politeia and that the idea of the basic interrelatedness of phenomena of different levels of collectivity constitutes a recurring topic through the history of political thought. It would take us too far to even attempt an abridged synopsis of this variegated subject as it reappears in the thinking of mankind. To refer the reader to acknowledged texts on the history of sociology would be tantamount to irritating him, for the subject transcends sociology in several dimensions, including logic, epistemology, ontology and a multitude of linguistic, psychological and statistical problem areas.

These remarks are not meant to be helpful, but rather to prepare the reader to accept a narrow and technical view of the problem of contextuality in sociological research as a useful way to tackle the more ramified extensions of the argument and to refrain from expecting anything from diffuse rhetoric about "complexity" and the salvation flowing from "reduction". A discussion of multi-level and cross-level-analysis in terms of elementary statistics might not only favor elucidation but also structure the consequential questions of a less technical character.
This is the more to be advertised as empirical research holds the majority among those asking for clarification of the multi-level issue. The frequent cry for theory-development as a panacea also for these particular ailments may be more than a token of intellectual fatigue of the uninitiated, but defining units, forming stages in sampling, weighting and re-weighting strata, estimating parameters as all other acts in empirical practice contain something very alien to theory formation and intra-syntactic juggling of concepts, in fact they are "naive" relative to "pure" thought as any action necessarily is whilst mere verbalizing of thoughts must seem etherial and irresponsible to the working class to whom CICOUREL compared empirical labor.

\section{Elements: units, dimensions, variables}

In the language of research variables are measurable properties the extensions of which are associated to each of a set of units. A variable $X$ may have size $x_{i}$ for an individual $i(i=1,2, \ldots$ $\mathrm{n})$. If this size varies over time we might measure $x_{i t}$ at some period or point $t$. $i$ and $t$ are then dimensions of units or, in terms of sampling theory, types of realizations, since $\mathrm{x}_{\mathrm{it}}$ constitu- 
tes a special realization of a random variable $X$. $i$ and $t$ have been randomly selected out of two universes, viz. the universe of individuals and the universe of time periods. This, however, is only one particular interpretation. $t$ may be one unit of a set of adjacent units whithin an interval between $t_{0}$ and $t_{k}$. $x_{i t}$ is then a time series with discrete time intervals for individual $i$. In a system of difference equations $x_{i t}$ may be written as an explicit function of the variable $t$ : $x_{i t}=f_{i}(t)$. Here $t$ can be discrete or continuous. The function $f_{i}()$ contains certain parameters which may be known or estimated so that for each $i x_{i t}$ can be predicted or postdicted. That is, the history of $i$ with respect to $X$ is known or short: the $\mathrm{X}$-history of $\mathrm{i}$ is described by $\mathrm{x}_{\mathrm{it}}$.

Why can't we treat $i$ in the same fashion? That would mean finding a general function $f(i, t)$ $=x_{i t}$ of two variables. This is out of question since i can never become a variable. Individuals can only be enumerated, not measured (without explicitly introducing variables characterizing individuals). Accordingly the "assessment of man" is, strictly speaking, just a figure of speech, the correct title being "The assessment of variables associated with individuals". Let us extend the dimensionality of $x_{i t}$ to $x_{i t r}, r$ being the index of a region. Now there are 3 dimensions of $x, t$, which can be made a variable and $i$ and $r$ which can not. However, as in physics, $r$ could be replaced by special coordinates $r_{1}, r_{2}$ and $r_{3}$ which again could be treated as discrete or continuous variables. Thus, $x_{i t r}=f_{i}\left(t, r_{1}, r_{2}, r_{3}\right)$ would express the world line of individual $i$, with respect to $X$.

The simple notation $x_{i t r}$ covers up the heterogeneity of the nature of the dimensions. Turning $t$ and $r$ into variables means going into the direction of nomothetic explanation while treating them as indices of dimensions of units means sticking to ideographic description of data. The fact that $i$ cannot be considered a variable ties us down to individualistic explanation, $f_{i}$ being specific for $i$. Sociology is trying to liberate the $f_{i}$ 's from the $i$ 's. One way is to consider groups to which the $i$ 's belong. If $g$ is a group-index, $\mathrm{X}_{\text {igtr }}$ has gained another dimension, but $\mathrm{X}$ is still an individual property.

\section{Variability of and over dimensions}

If we want to explain the behavior of a variable $y$ in terms of a variable $x$ both have to vary in at least one common dimension. The dimension selected determines the interpretation of the coefficients estimated. $Y_{i}=a+b x_{i}+u_{i}$ contains $a$ and $b$ as parameters the estimate of which over individual variations (over i) limits us to the interpretation that a positive $b$ shows that whenever an individual has a high value in $X$ it does so also with respect to $Y$ and vice versa. The equation $\mathrm{y}_{\mathrm{t}}=\mathrm{k}+\mathrm{cx}_{\mathrm{t}}+\mathrm{v}_{\mathrm{t}}$ means something quite different, even if by estimation over $t$ we would obtain $a=k$ and $b=c$. If TAYLOR (1968: 120) writes, "in my use of aggregate data to determine the relationship between social change and international aggression, I have found, quite unexpectedly, that there is considerable empirical similarity between the results of both kinds of analysis. With only a few exceptions the conclusions of longitudinal analysis have been identical with those of cross-sectional analysis. Since it is cheaper and simpler ...", he in fact identifies interpretation of $b$ and $c$ because of their similar order of magnitude ( $\mathrm{i}=$ individual country in his case). Mixing up dimensions in this way may be "cheaper and simpler" but TAYLOR himself implies this procedure to be inaccurate by continuing "But when a substantial body of theory already has been formulated, as on political development, only accurate answers can advance the field". The index $t$ replacing $i$ or $r$ in the same variables and the same equations change a static into a dynamic substantive theory as $\mathbf{g}$ for $\mathbf{i}$ makes a collectivistic theory out of an individualistic one. International comparison may be considered just an estimation strategy as evolutionism, but theoretically they are no alternatives, but complementary.

The background of estimating parameters is the comparison of variations over one dimension keeping the others constant (as in partial correlation or partial differentiation the other variables are kept constant). Thus interrelations (and their parameters) between $x_{i t}, y_{i t}$ and $z_{i t}$ can be estimated by either keeping $i$ constant and varying $\mathrm{x}, \mathrm{y}$ and $\mathrm{z}$ over $\mathrm{t}$ or the other way around. In the first case we find "laws" about individual $i$, differing among different individuals, in the latter case we are discovering interrelations ("laws") which all individuals $i=1,2, \ldots . n$ 
have in common at time $t$. In the simple case of $y_{i t}=a x_{i t} a$ is of zero-dimensionality, i.e. a constant with respect to all dimensions. Keeping $\mathbf{i}$ fixed and estimating a via $\mathbf{t}$ forces us to make a variable in one dimension, viz. $i$, so that the $a_{i}$ 's take the place of just one $a, a_{i}$ being no constant with respect to $i$, but still with respect to $t$. Would we add yet another dimension, viz. $t$, to $a_{i}$ and arrive at $y_{i t}, x_{i t}$, no constancy would be left in a relative to $y$ and $x$ and a could not be estimated: Instead, $a_{i t}=$ $\mathrm{y}_{\mathrm{it}} / \mathrm{x}_{\mathrm{it}}$ would always hold, a tautological repetition of the original equation. Only by introducing a third dimension $k$ to $y$ and $x$ could we estimate all the $a_{i t}$ 's in $y_{i t k}=a_{i t} \cdot x_{i t k}$.

This is just a simple demonstration of the fact that parameters or explaining agents have to be of at least one dimension (in variability) less than the "variables" whose interrelations are to be reflected in those parameters.

Having arrived at all the $a_{i}$ 's as explained we can study the variation of this variable of one dimension. If we have $b_{i}$ 's from another equation a third equation can be set up explaining relations between the $a_{i}$ and $b_{i}: a_{i}=k b_{i}+v_{i} \cdot k$ measures the influence of w's influence on $z$ on $x$ 's influence on $y$, if $y_{i t}=a_{i} \cdot x_{i t}+u_{i t}$ and $z_{i t}$ $=b_{i} \cdot w_{i t}+e_{i t}$ and can be estimated via $i$.

In a similar way $a_{t}$ could be estimated via $i$ in $y_{i t}=a_{t} \cdot x_{i t}$. However, following our argument above, $a_{t}$ may be considered a time function in its own right, with parameters expressing some temporal regularity or "historical law" as f. i. WAGNER's law that national budgets tend to increase as a fraction of GNP over time. If on the other hand $a_{t}$ is treated just as a time series or a variable in a static theory (equation or system of equations), then $t$ disappears or is used up in the estimation process (paralleled) in the explanatory act.

Finally, realizations of the variables can be used simultaneously in all dimensions (via all $i$ and $t$ ). A single index $j$ would suffice enumerating all i's and t's in any order. This would mean to estimate a constant $a$ in $y_{i t}=a \cdot x_{i t}+u_{i t}$ or $y_{j}=a x_{j}+u_{j}$.

\section{Hierarchical dependence of dimensions}

In multi-level analyses dimensions are not independent of each other any more as $i$ and $t$ were in the last section. Take $r$ as some context to which $i$ belongs, $i$ belonging to no other $r$. Then $x_{i r}$ can vary within $r$ (or keeping $r$ constant) and over all i's belonging to $r$, but it cannot vary over $r$ keeping $i$ fixed, because a specific $i$ is lost by a switch from one $r$ to the next. For each variable $X$ there exists a data matrix $\left(x_{i r}\right)$ with $(i=1,2, \ldots n, r=1,2, \ldots k)$ $n$ k cells but only $n$ entries, $n(k-1)$ cells being empty.

This simple fact that a concrete individual has no intercontextuality and therefore cannot display variations in any variables over regions, organizations, groups or any other unit of higher levels of collectivity raises the crucial question of the whole micro-macro issue: how can the impact of contexts (societies, groups etc.) on individuals be measured at all? For a more general formulation let $r_{0}=i, r_{1}=$ context one level above the individual, $r_{2}=$ two levels above $i$, $\ldots$. Each $r_{p}$ belongs to exactly one $r_{p}+1$ $(p=0,1, \ldots k)$ with $k$ contexts being considered, i.e. we have a hierarchical structure of dimensions, shortly a $\mathbf{h}$ - dimensionality.

How can cross-level effects then be estimated?

In order to further analyse the problem we take the simple form $y_{i r}=a x_{r}+u_{i r}$. The contextual variable $x_{r}$ is influencing the individual-contextual variable $y_{i r}$, and a is measuring this influence. Estimating a via $r$ is impossible, since no sum of $y_{i r} \cdot x_{r}$ can be formed. Going via $i$ results in the estimate $\hat{a}=\bar{y}_{\mathrm{r}} / \mathrm{x}_{\mathrm{r}}$ and $\mathrm{y}_{\mathrm{ir}}=\bar{y}_{\mathrm{r}}$ is made a constant relative to $i$. So individual variation is eliminated ex post. The last and third way is to treat $x_{r}$ as an individual variable and summing over all $i$ and $r$. The number of elements $i$ in $r$ being $n_{r}$ this sum would be

$$
\sum_{\mathrm{r}} \sum_{\mathrm{i}} \mathrm{y}_{\text {ir }} \mathrm{x}_{\mathrm{r}}=\sum_{\mathrm{r}} \mathrm{n}_{\mathrm{r}} \overline{\mathrm{y}}_{\mathrm{r}} \mathrm{x}_{\mathrm{r}}
$$

for the numerator and

$$
\sum_{r} \sum_{i} x_{r}^{2}=\sum_{r} n_{r} x_{r}^{2}
$$

for the denominator of the estimate for a. We see that the weight or size of the context enters 
the estimate and might warp it considerably. In addition, dimensions are confounded this way. What really happens is that a contextual variable is modelled as an individual one. This is usually the case in survey research when the size of the community of the informant is used as an explanation of his individual behavior. But here size is made an explicit variable while in the estimation procedure above it is only implicit.

The objection here is against hidden compositional effects. In a country where 80 p.c. of the population live in big cities bigness or urbanization seems to matter much more than in a country with only 30 p.c. living there although the impact of urbanization on individuals may be much stronger in the latter case. The matter boils down to weighted vs. non-weighted averages (see KISH 1965). In the three or more level case the problem is aggravated. Only if all $n_{r}$ are the same size no problem comes up.

\section{Eliminating size effects}

The artificial character of parameters estimated on the basis of frequency of occurence is apparent quite distinct from contextual analysis. In bivariate coefficients as f.i. phi the co-incidence of values of two variables determines their order of magnitude. Mere size effects are then carried over to chi-square testing and similar statistical decisions. In ANOVA-procedures this question is clearly seen. An experimental design ${ }^{1}$ with $m$ independent dichotomous variables $x_{j}(j=1$, $2, \ldots m)$ and a metrical dependent variable $z_{i}$ with $\mathrm{i}=$ index of the $2^{\mathrm{m}}$ configurations possible with $m$ variables $x_{j}$ will provide for an elimation of $n_{i}$ as the number of units in each configuration in the model.

$z_{i}=a_{1} x_{i 1}+a_{2} x_{i 2}+\ldots+a_{m} x_{i m}+u_{i}$

$\left(\mathrm{x}_{\mathrm{ij}}=+1\right.$ or $-1, \mathrm{i}=1,2, \ldots 2^{\mathrm{m}}, \mathrm{a}_{\mathrm{j}}=$ constants $)$.

$\mathrm{X}=\left(\mathrm{x}_{\mathrm{ij}}\right)$ is an orthogonal matrix, each row of which refers to one configuration or i-type, not to one individual. Using least squares minimization on $u_{i}$ the estimates $\hat{a}_{j}$ are free from $n_{j}$, the number of realizations or units in the respective i-type. $a_{j}{ }^{2}$ measures that part of $\operatorname{Var}(z)$ attributable to $x_{j}$.

1 For details of this model see HARDER 1969.
Defining i-types in this way and not working with individuals any more solves the problem of non-intercontextuality of individuals in multi-level-analysis, because context-variation and comparison across i-types is possible while across individuals it was not. In this respect the new $i$ has the same property as $t$ in our first reasoning: $i$ and $r$ (context) are now independent dimensions and regression models can be set up without running into the difficulties of the last section.

\section{Estimating individual from ecological parameters}

A special way to bridge the gap between microand macro-levels of analysis is the estimation of individual from ecological or contextual parameters. The simplest equation connecting both levels is:

$$
\text { 1) } z_{\text {ir }}=a x_{i r}+b y_{r}+c x_{i r} y_{r}+u_{i r}
$$

$\left(Z_{i r}\right.$ and $x_{i r}$ are dichotomous variables,

$y_{r}=$ average of $x_{i r}=\frac{1}{n_{r}} \sum_{i}^{n_{r}} x_{i r}$ )

Summation within $\mathbf{r}$ over $\mathbf{i}$ (individuals within context $r)$ results in $\left(\sum_{i} u_{i r}=0\right)$ :

2) $w_{r}=a y_{r}+b y_{r}+c y_{r}^{2}$

If we are confronted with the general ecological equation

$$
w_{r}=g_{0}+g_{1} y_{r}+g_{2} y_{r}^{2}
$$

we have $a+b=g_{1}, g_{0}=o$ and $g_{2}=c$

and a resp. b cannot be identified.

This fact is sometimes taken as a general proof that nothing individual can be inferred from ecological relations. But this is just a consequence of setting $Z_{i r}=1$ or 0 (the same for $x_{i r}$ ). However, taking -1 instead of $o$ we arrive at the averages (for $Z_{i r}$ and $x_{i r}$ )

$$
2 w_{r}-1 \text { and } 2 y_{r}-1
$$

instead of $w_{r}$ and $y_{r}$ 
and at

4)

$$
\begin{aligned}
& 2 w_{r}-1=a\left(2 y_{r}-1\right)+b y_{r}+c y_{r} \\
& \left(2 y_{r}-1\right)
\end{aligned}
$$

or

5)

$$
\mathrm{w}_{\mathrm{r}}=.5\left(1-\mathrm{a}+(2 \mathrm{a}+\mathrm{b}-\mathrm{c}) \mathrm{y}_{\mathrm{r}}+\mathrm{cy}_{\mathrm{r}}^{2}\right)
$$

By comparing coefficients in 3) and 5) we obtain

$$
\begin{aligned}
& \mathrm{g}_{\mathrm{o}}=.5(1-\mathrm{a}) \\
& \mathrm{g}_{1}=.5(2 \mathrm{a}+\mathrm{a}-\mathrm{c}) \\
& \mathrm{g}_{2}=\mathrm{c}
\end{aligned}
$$

and the solutions (individual parameters):

$$
\begin{aligned}
& a=1-2 g_{o} \\
& b=-2+4 g_{o}+2 g 1+g_{2} \\
& c=g_{2}
\end{aligned}
$$

This solution shows that individual parameters can be inferred from ecological parameters if we use a suitable way of dichotomization. The problem really is in the accuracy of the estimates for the g's. Opening the model in the direction of multivariate analysis engenders all the troubles known to the regression analyst, but the solution can be extended. If in estimating the g's in (3) we give equal weight (preferably 1) to each $\mathrm{r}$, we cannot expect the triple $\mathrm{a}, \mathrm{b}$, and $\mathrm{c}$ deduced from this estimates to equal those $a, b$ and $c$ estimates derived directly from (1) by going via all $i$ and $r$, unless all $n_{r}$ are equal, this just being a necessary but by no means a sufficient condition for the equality of estimates.

The general character of inter-level inferences of the kind demonstrated can be indicated by the diagram ${ }^{2}$ :

$$
\text { explanantia }
$$

explananda

micro

macro

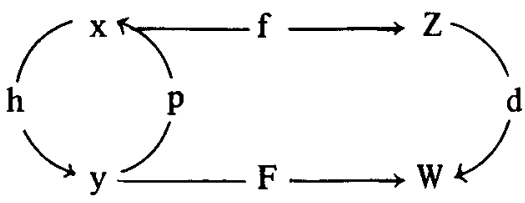

$f$ stands for the micro-theory, $F$ for some macrotheory, $h$ and $d$ for aggregation of macro-variab-

2 The diagram was used by FELS/TINTNER (1967: 71). les from micro-variables and $p$ for the intra-explanantia explanation of micro- in terms of macrovariables (macro-micro explanation). Aggregation by analytical procedures like summation of course does not constitute explanation.

What we did achieve by expressing $a, b$ and $c$ in terms of the g's was going from $F$ to $f$ in the above diagram, i.e. deducing micro- from macrorelations.

Sociologists believing in the principal reducibility of macro- to micro-properties and hypotheses might argue that the g's are also to be deduced from $\mathbf{a}, \mathrm{b}$ and $\mathrm{c}$. Furthermore, they will interprete the diagram as being commutative, i.e. $\mathrm{w}=\mathrm{d}(\mathrm{z})=\mathrm{d}(\mathrm{f}(\mathrm{x}))=\mathrm{F}(\mathrm{y})=\mathrm{F}(\mathrm{h}(\mathrm{x}))$. But this would mean ignoring the fact that $Z_{i r}$ as an individual variable depends on $y_{r}$ (a contextual one) in (1). Equation (1) is not represented by $f$ in the diagram, but by a morphism more complicated combining $\mathrm{x}$ and $\mathrm{y}$ and connecting this combination with $Z$. $x=p(y)$ is one indirect expression of fact which would lead to some ecological relation like (3).

The context effect signalled by some $\mathrm{c}$ significantly different from o and giving expression to effective interaction between individual $\left(\mathrm{x}_{\mathrm{ir}}\right)$ and contextual $\left(\mathrm{y}_{\mathrm{r}}\right)$ variables suffers from the deficiency criticized in the beginining: some $c=g_{2} \neq 0$ does not represent a theory but at best an empirical discovery (in the absence of compositional or other artefacts) which we cannot understand, because $y_{r}$ does not co-vary with $Z_{i r}$. The basic fact is that $i$ cannot be transferred from one context to the next. Introducing i-types as suggested does away with this problem, but the theoretical issue remains, how to define equivalence of such types across different contexts.

\section{Dynamics of individual mobility}

The most elementary theoretical idea coming to grips with intercontextual comparison of individuals is mobility. An act of mobility (horizontal, vertical or any other way) could be defined ${ }^{3}$ as switching contexts: moving from one job to another, changing the place of residence, the

3 An integrated view of contextual aspects of mobility can be found in HARDER (1973: 105). 
affiliation to some group or person etc. ... . Assuming the individual staying identical while moving (i.e. successively "belonging to") through diverse environments we would have a quasi-experimental condition for comparing the influence of these environmental variation on his behavior, belief-systems and orientations. But what would identity mean? Would staying in the same i-type be enough? Or, more fundamentally: would that be possible? Our definition of a social category as a configuration (i-type) of variables $x_{j}$ depends of course on the $x_{j}$ 's selected as independent variables. Accompanying somebody leaving college and entering some government post (as a regression analyst) would confront the participant observer or data collector with changes on both sides of the equation, if professional status is considered to be included among the $x_{j}$ 's. On the left-hand side we might have some conservatism-leftism scale for the dependent variable. The observed individual (hopefully or not) might change from liberal to reactionary while switching his status ${ }^{4}$. The theory has to specify how his moving changes his identity, how the new context comes in, what the influence of leaving the old one is as compared to anticipations of the new one (memory push and anticipation pull) and other aspects. This scratch at the surface of the mobility complex should warn the multi-level analyst engaged in dynamic theorizing, in empirical socialization or mobility studies, because "it is going to be worse before it is going to be better".

It seems quite clear that static pathmodeling would be no help. The suggestion of cohort analysis comes naturally, but the support from it would be substantial unless a theory could be formed comprising an explanation of historical (secular) change of mobility patterns in the relevant professional field, the determination of political attitudes and their dynamics over the life-cycle and some theory of life strategies and orientation schemes. These notions being obvious and somewhat arbitrary the real test comes when

\section{A German research team at Konstanz has found} considerable evidence for the proposition that university students turn more liberal after leaving highschool and return to a more conservative political attitude after entering professional life, a phenomenon called the "Konstanzer Wanne" (tub). we start collecting or asking for data. Dynamic theories and models are all well-meant, but of little value if we have to delve into well-established historical research with biographies, diaries, letters and similar types of data at our disposal. The more obstrusive measure as penetrating into the deep past of individuals by interviews are severely underdeveloped. Development of this tool might prove very useful for the purpose of contextual analysis of mobility and change, which in turn is a prerequisite to any answer to the challenge of multi-level analysis. This challenge is not an outcome of casual methodological predilections of some sociologists, but it is aiming at the very core of sociological endeavor, viz. to explain social facts in terms of (DURKHEIM's) social facts and going beyond that in integrating individualistic theories of anthropology resp. biology.

The diagram in the last section and the rather elementary model do suggest that micro-macro and macro-micro explanations are no alternatives, but partially just different aspect of the same story. The macro-macro type of explanations has its main field in historical (empirical) sociology or some structural-functionalist theorizing. As GALTUNG has suggested, socio-structural change might today transmit its pressure down to the lowest level of the individual person. This is in no way the only admission that individuality and personal variables have been neglected in macro-sociological theory formation and for many reasons anthropology should re-enter the scene, not necessarily in the version of the "homo Homanianus"s.

\section{Bibliography}

ALKER, H. R., 1965: Mathematics and Politics. Collier-Mac-Millan, New York and London.

ALKER, H. R., 1969: A Typology of Ecological Fallacies, in: Dogan and Rokkan (eds.), Quantitative Ecological Analysis in the Social Sciences. MIT-Press, Cambridge and London, 69-86.

ARROW, K. J., 1963: Social Choice and Individual Values. John Wiley, New York (2nd ed.).

5 G. C. HOMANS' "Bringing Man Back In" (1964) has been countered four years later by ALLEN $H$. BARTON's "Bringing Society Back In" (1968), where the problems in multi-level analysis are analyzed with special emphasis on perception. 
BARTON, A. H., 1968: Bringing Society Back, in: Survey Research and Macro-Methodology, in: American Behavioral Scientist, vol. XII,2,1-9. BLAU, P. M., 1961 : Structural Effects, in: American Sociological Review, vol. 25, 178-193.

DAVIS, J. A., J. L. SPAETH, C. HUSON, 1961: A Technique for Analyzing the Effects of Group Composition, in: American Sociological Review, vol. 26, 215-225.

DUNCAN, O. D., R. P. CUZZORT, B. DUNCAN, 1961: Statistical Geography. The Free Press of Glencoe, N.Y.

EBERLEIN, G., 1971: Theoretische Soziologie heute. Stuttgart: Enke.

FELS, E., G. TINTNER, 1967: Methodik der Wirtschaftswissenschaften, in: Manfred Thiel (ed.), Methoden der Sozialwissenschaften. Munich and Vienna.

GOODMAN, L. A., 1959: Some Alternatives to Ecological Correlation. American Journal of Sociology, vol. LXIV, 610-625.

HARDER, Th., F.-U. PAPPI, 1969: Multiple-Level Regression-Analysis of Survey and Ecological Data, in: Soc. Sc. Information 8(5), 43-67.

HARDER, Th., 1969: Model Construction in Multivariate Multi-Level Analysis, in: Quality and Quantity $1 / 2,153-167$.

HARDER, Th., 1973: Dy namische Modelle in der empirischen Sozialforschung. Stuttgart.

HUMMELL, H.-J., K.-D. OPP, 1968: Sociology without Sociology, Inquiry, vol. 11.

HOMANS, G. C., 1964: Bringing Man Back in, in: ASR 29, 809ff.

KISH, L., 1965: Sampling Organizations and Groups of Unequal Sizes. ASR 30,2, 564-572.
LAZARSFELD, P. F., 1959: Methodological Problems in Empirical Research. In: Transaction of the Fourth World Congress of Sociology, vol. II, London, 225249.

LINZ, J. J., 1969: Ecological Analysis and Survey Research, in: Dogan and Rokkan (eds;), Quantitative Ecological Analysis in the Social Sciences. MIT-Press, Cambridge and London, 91-131.

SCHEUCH, E. K., 1967: Society as Context in CrossCultural Comparisons, in: Social Science Information, vol. VI,5, 7-23.

SCHEUCH, E. K., 1969: Social Context and Individual Behavior. In: Dogan and Rokkan (eds.), Quantitative Ecological Analysis in the Social Sciences. MIT-Press, Cambridge and London, 133-155.

SCHUESSLER, K., 1969: Covariance Analysis in Sociological Research. In: Edgar F. Borgatta (ed.), Sociological Methodology. Jossey-Bass Inc., San Francisco.

SELVIN, H. C., 1958: Durkheim's Suicide and Problems of Empirical Research. American Journal of Sociology, vol. 63, 607-619.

TAYLOR, Ch. L., 1968: Further Problems: A Consideration of Other Views. In: Ch. L. Taylor (ed.), Aggregate Data Analysis. Mouton, Paris, 115-138.

VALKONEN, T., 1969: Individual and Structural Effects in Ecological Research. In: Dogan and Rokkan (eds.), Quantitative Ecological Analysis in the Social Sciences. MIT-Press, Cambridge and London, 53-68.

Anschrift des Verfassers:

Prof. Dr. THEODOR HARDER

48 Bielefeld, Universität 